\title{
Conductive Carbon Filled Polymeric Electrodes: Novel Ion Optical Elements for Time-of-Flight Mass Spectrometers
}

\author{
Matthew F. Appel and Wytze E. van der Veer \\ Department of Chemistry, University of California, Irvine, California, USA \\ Thorsten Benter \\ Bergische Universität GH Wuppertal, Wuppertal, Germany
}

\begin{abstract}
A new technique employing conductive polymeric electrodes for the generation of linear electric fields is proposed and validated. A hollow cylinder comprised of DuPont's Vespel SP-22, a polymer loaded with conductive carbon particles, is used to enhance the performance of a hybrid reflectron compared to a conventional dual stage gridless reflectron composed of discrete metal ring electrodes. Both devices have comparable physical dimensions and nearly identical ion optical properties. Instrumental resolution measurements are used to validate this novel design. (J Am Soc Mass Spectrom 2002, 13, 1170-1175) (C) 2002 American Society for Mass Spectrometry
\end{abstract}

$\mathrm{T}$ The control and use of charged particle beams is of considerable interest to scientists in a wide range of fields. Chemists, physicists, and biologists have all benefited from the use of these beams to probe new materials, control the momentum of interacting particles and analyze elements within complex mixtures [1,2]. Imperative to all of these applications is the manipulation of the momentum of charged particles via precisely aligned electrical fields, which are generally created from discrete electrode stacks. Typically, these electrodes are produced from metallic materials, e.g., stainless steel. However, researchers have recently begun to use novel, conductive materials to generate electrostatic fields [3,4]. One of the disciplines that has made use of these new materials is mass spectrometry, particularly time-of-flight mass spectrometry (TOF MS).

In general, TOF instruments utilize a series of metal rings (electrodes) connected via bleeder networks of resistors to approximate linear fields. Combinations of these linear fields are then used to generate electrostatic lenses as well as multiple stage ion sources and reflecting devices (reflectrons). Recently, it has been proposed to combine electrodes and resistors into a bulk conductive electrode. In the late 80 's, Gruen et al. filed a patent for a new photo-ion spectrometer [3]. This instrument utilized an electrostatic analyzer, which used a plate

Published online August 22, 2002

Address reprint requests to Dr. W. E. Van Der Veer, Department of Chemistry, University of California, Irvine, CA 92697-2025. E-mail: wevdveer@uci.edu composed of a resistive thick film deposited as paste on an insulator surface. It was reported that screen-printing BIROX (DuPont, Wilmington, DE), a resistive bismuth-ruthenium oxide compound, on top of a conductive paste allowed the generation of precise electrostatic fields and was ideally suited to the creation of irregularly shaped and non-linear potentials. Even and Dick have introduced a similar treatment [4]. Here, an ion source with three acceleration regions was fabricated from Alumina ceramic tubes brush coated with a resistive paste. Once coated, the cylinders were air fired to create a vacuum compatible conductive surface. When coupled to a $700 \mathrm{~mm}$ long flight tube, this linear instrument provided a mass resolution of $\mathrm{m} / \Delta \mathrm{m}=$ 1200 (FWHM).

In an effort to create increasingly compact TOF instruments, our research group has focused on novel approaches to instrument design and laser ionization techniques [5-7]. With this in mind, we have initiated research that is concerned with the production of "ide$\mathrm{al}^{\prime \prime}$ electrostatic fields. We have focused on the application of novel non-metallic surface and volume conductive polymers for the generation of these fields. Unlike the screen-printing and brush coating methods of conductive electrode design, these conductive electrodes are particularly attractive as the electric field strength can be precisely tuned simply by machining. In this paper, we report on the use of Vespel SP-22 (DuPont), a polyimide loaded with carbon black, as a novel surface conductive electrode for application with mass spectrometry. In order to test the viability of Vespel as a new 


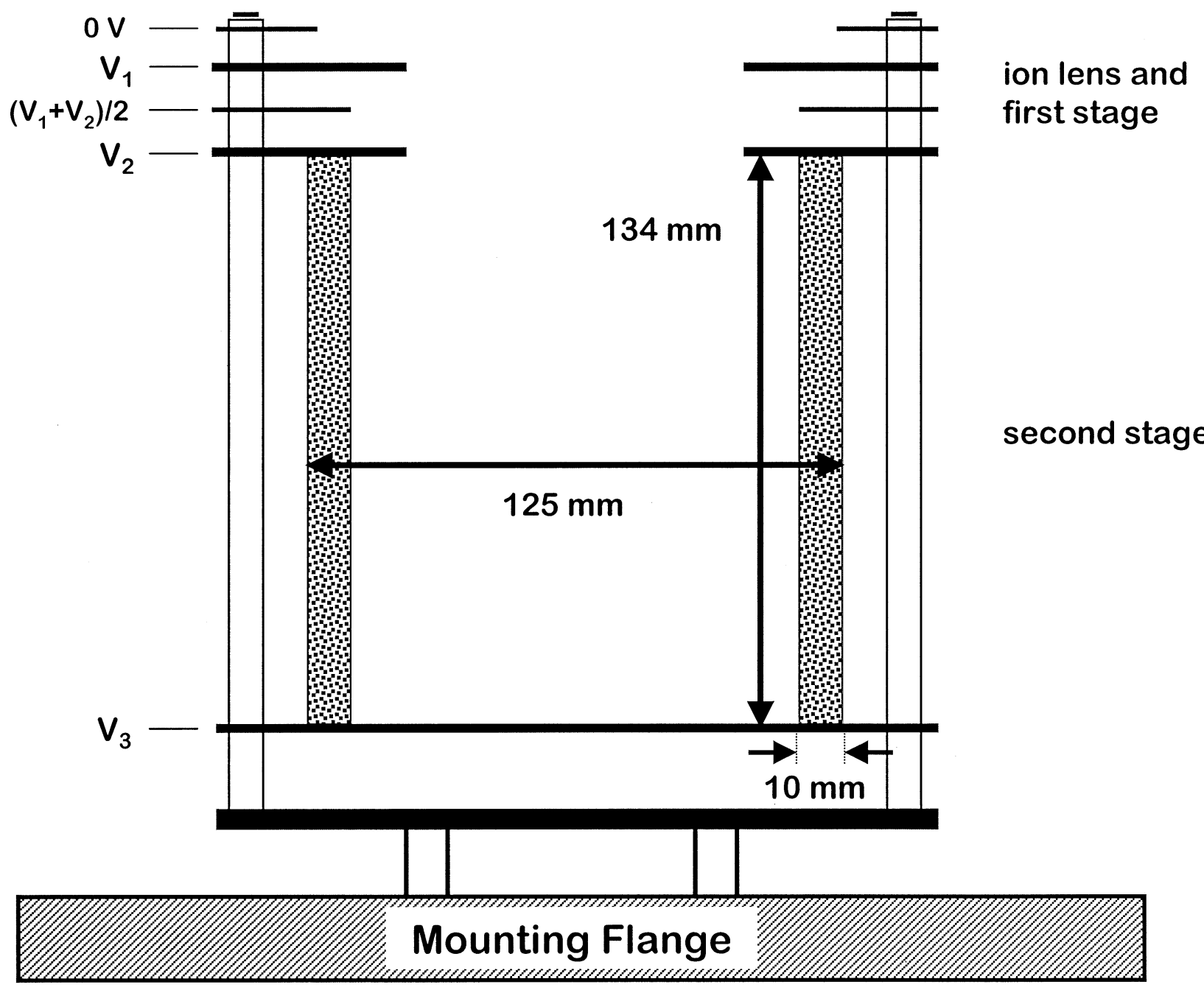

Figure 1. Schematic representation of the hybrid polymeric/stainless steel reflectron. The first stage incorporates traditional stainless steel ring elements (black lines) coupled via resistors. The second stage (dotted areas) is composed of a single hollow cylinder of Vespel SP-22. White areas indicate electrically isolating material. Cf. Figure 2 for a description of the potentials applied.

material for generation of linear electric fields, we have replaced the second stage of a commercial dual stage gridless reflectron with a solid segment of Vespel SP-22. We describe the results obtained with this hybrid reflectron with respect to effects on mass resolution of an existing Bruker TOF1 mass spectrometer.

\section{Experimental}

\section{Electrode Design}

Conductive polymeric materials can be both intrinsically conducting, i.e., polymers that possess extended conjugation and the resulting overlap of $\pi$-electrons, and intrinsically insulating, i.e., polymers that contain immiscible conducting particles, for example metal powder or graphite. Both types of materials have found a variety of applications; these include packaging materials for devices sensitive to static electricity, corrosion inhibitors and chemical and biological sensors $[8,9]$, as well as self-lubricating bearings or ferrule material in gas chromatography. We focus on the application of polymer blends with conductive fillings as resistive ion optical electrodes.

When selecting a polymeric bulk conductive electrode material for ion optical purposes, it is imperative that the polymer is stable in a high vacuum environment, i.e., does not outgas, is chemically inert, and exhibits the required electrical properties. Upon inspection of the commercially available conductive polymer blends, the DuPont line of Vespel polymers was chosen. Due to their unique chemical and thermal properties, these polyimide-based materials are especially well suited for use in the high vacuum environment of mass spectrometers [10]. A classical application of polyimides is found in ion optical engineering as replacement material for the comparably expensive ceramic spacers used as insulators in electrode stacks [11].

Polyimides usually contain both electron donating 


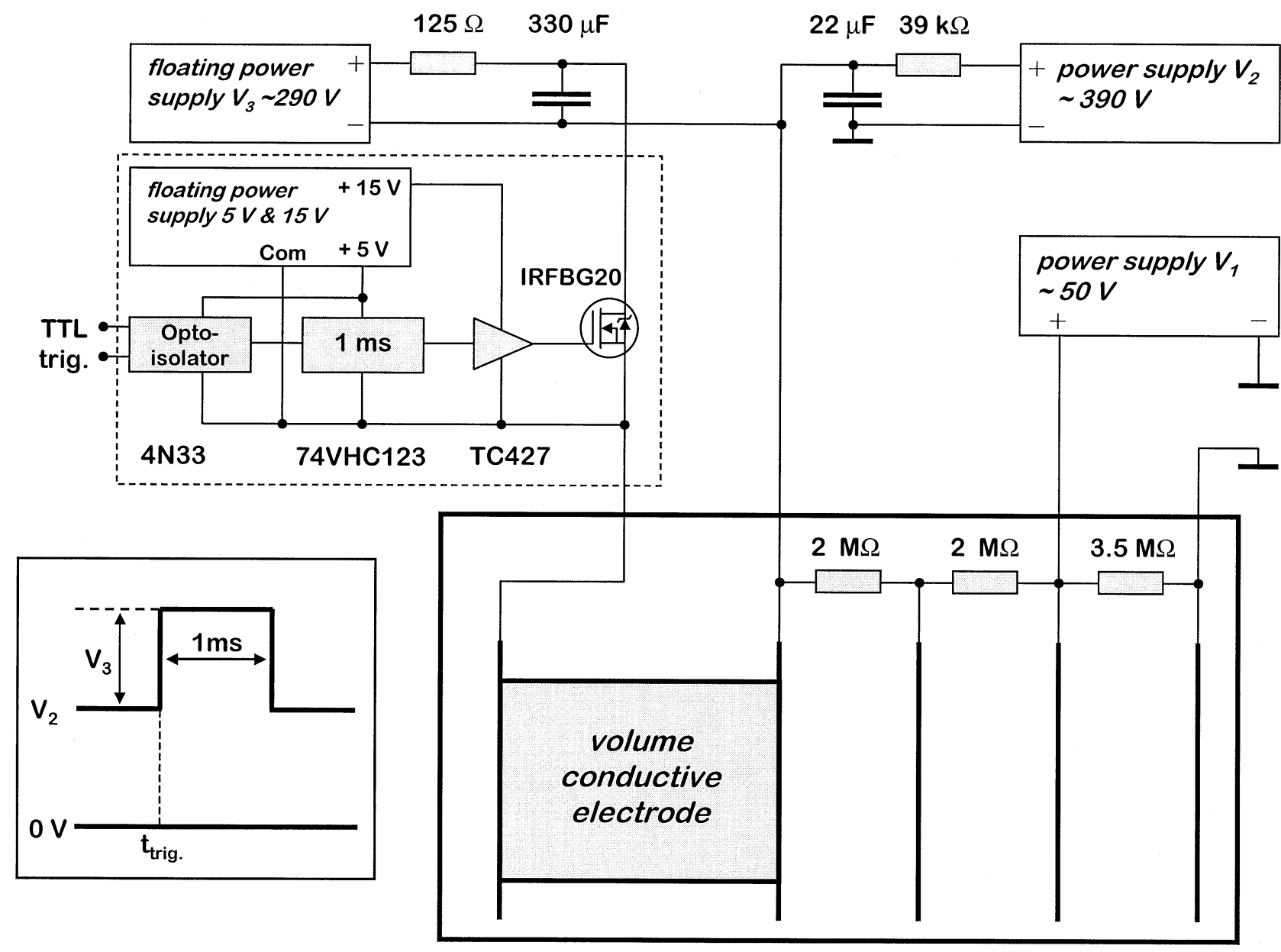

Figure 2. Diagram of the pulsing electronics driving the polymeric reflectron. The voltage difference across the volume conductive electrode was maintained at 0 volts and pulsed to $V_{3}$ for $1 \mathrm{~ms}$ centered around each laser shot (see inset).

and withdrawing groups, facilitating the formation of robust charge transfer complexes both within individual units of the polymer and between adjacent chains [12]. As a result, they are exceptionally strong and relatively non-porous, which limits chemical uptake and minimizes the degree of outgassing $[11,13,14]$.

DuPont's Vespel polymers can be obtained with a wide range of graphite filler loadings. During mixing, the conductive characteristics of these polymers can be mechanically adjusted through the choice and loading of filler. Polymers with a large concentration of graphite can be highly conductive [15]. Depending on the proximity of the carbon particles on/in the insulative substrate, electrons tunnel and/or hop from aggregate to aggregate [16]. As the volume percent of carbon is increased or decreased, the material will exhibit a gradual change in resistivity [15]. When carbon powder is used as the conductive filler, as is the case with Vespel SP-22, machining exposes aggregates on the surface and the current is preferentially carried along the exterior of the electrode. In this case, the resistance is not affected by the thickness of the bulk material.

Using a hollow cylinder comprised of Vespel SP-22
( $40 \%$ by weight graphite), we replaced the resistive network of stainless steel electrodes from the second stage of a commercial Bruker (Bremen, Germany) TOF1 gridless dual stage reflectron. Overall, 12 resistively coupled stainless steel electrodes were exchanged for a $134 \mathrm{~mm}$ hollow cylinder of Vespel. Due to size constraints associated with the original segment of polyimide obtained from DuPont, the Bruker reflectron is replaced by a scaled down version of the original. Using the ion trajectory simulation program package SIMION 7.0 [17], it is determined that this reduction in size should have negligible impact on the mass resolution of spectra obtained with the size-altered instrument. A schematic representation of the hybrid reflectron can be seen in Figure 1.

The minimum resistance of the polymeric cylinder is limited by heating effects and the rating of the supply needed to deliver the current required to maintain the potential across the reflectron. Heating is a concern as elevated temperatures can influence the conductivity of the polymer material [16]. The maximum rating of the power supply providing the voltage to the reflectron is $15 \mathrm{~mA}$ at $1000 \mathrm{~V}$, limiting the minimum resistance to 


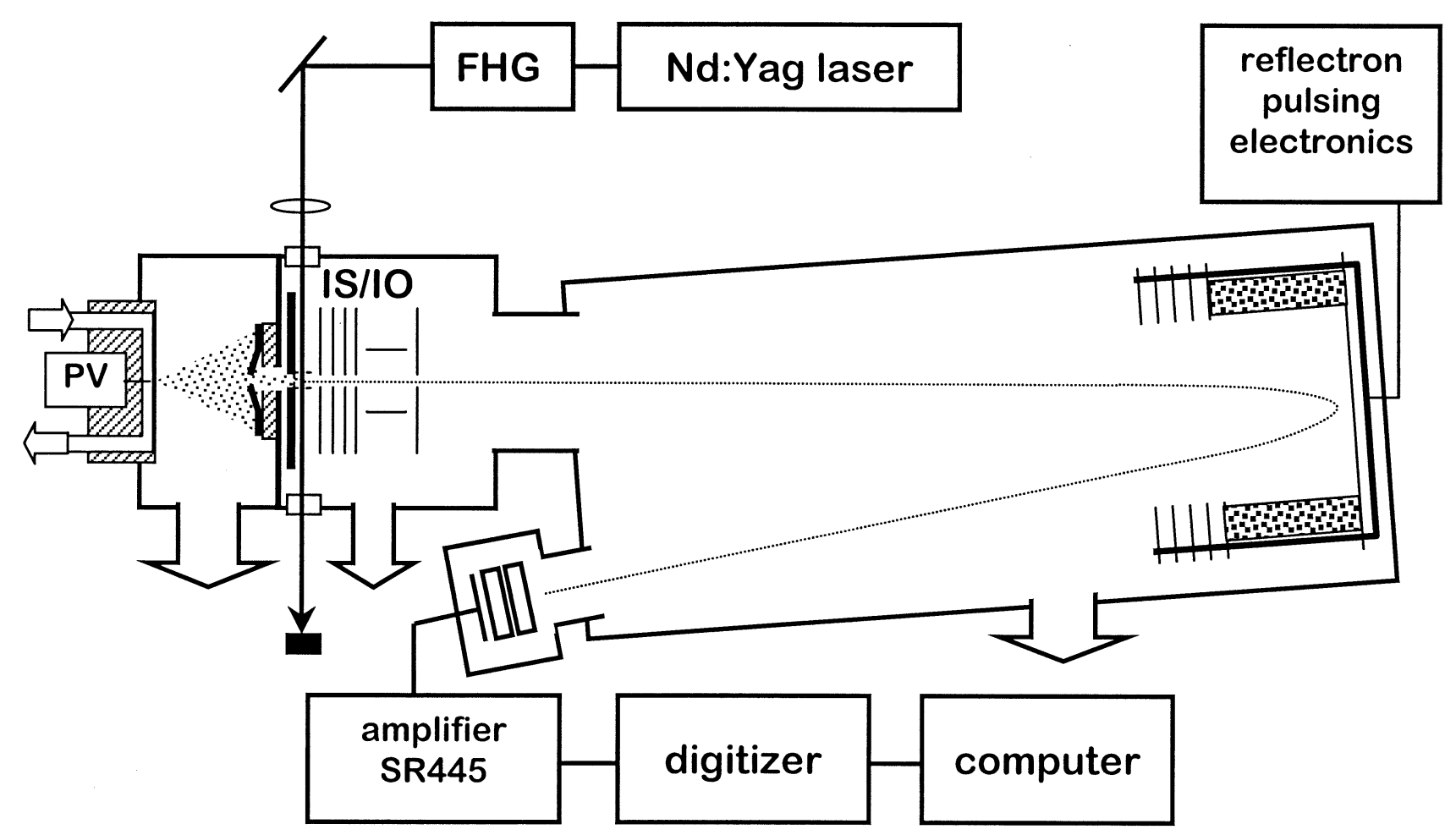

Figure 3. Schematic illustration of the experimental set-up. The Bruker TOF1 is shown with a home built differential pumping stage and hybrid polymeric/stainless steel reflectron. Photoionization occurs roughly $5 \mathrm{~cm}$ downstream of the pulsed valve. FHG = fourth harmonic generator; IS = ion source; $\mathrm{IO}=$ ion optics; $\mathrm{PV}=$ pulsed valve.

$\sim 70 \mathrm{k} \Omega$. In order to significantly reduce the average power requirement, in particular with respect to future applications in miniaturized TOF analyzers, the voltage across the reflectron was switched from zero to high potential with a duty cycle of 1:100. The present mass spectrometer is used in conjunction with a pulsed laser ionization source that operates at a repetition rate of $10 \mathrm{~Hz}$. The maximum transit time of ions with a mass $\mathrm{m}<1200 \mathrm{Da}$ from the ionization region to the detector is $\sim 250 \mu \mathrm{s}$. Switching the reflectron on for $1 \mathrm{~ms}$ centered on each laser shot allows ample time to stabilize the voltages. With this approach we are able to use a polymer cylinder machined to a length of $134 \mathrm{~mm}$ and a diameter of 125 $\mathrm{mm}$. The wall thickness is $10 \mathrm{~mm}$.

In Figure 2, the driving electronics of the switched hybrid reflectron system is depicted. The voltages $V_{1}$ and $V_{2}$ are provided by two independent power supplies, which are part of the original electronics of the Bruker TOF1 system and a SRS PS325, respectively. The reflectron voltage $\mathrm{V}_{3}$ is supplied by a Bertan (Hicksville, NY) 230-01F floating power supply. The current to the reflectron is switched by a IRFBG 20 MosFet, which is essentially a resistor with infinite resistance when off and $\sim 10 \Omega$ when on. The electronic circuit is floated to the end voltage of the reflectron. The circuit is triggered by a TTL pulse, which is galvanically decoupled from the high voltage circuit via an opto-coupler. The trigger fires a $1 \mathrm{~ms}$ monostable multivibrator and its output pulse is further amplified to drive the MosFet. The voltage $\mathrm{V}_{2}$ is stabilized using a capacitor, $\mathrm{C}=22 \mu \mathrm{F}$, minimizing interferences from the pulsed reflectron current. The voltage across the reflectron is stabilized within the pulse to $0.1 \%$ with a $330 \mu \mathrm{F}$ capacitor.

\section{Experimental Conditions}

Laser ionization experiments were performed using a Bruker TOF1 time of flight mass spectrometer fitted with a home-built differential pumping stage and pulsed sample inlet system, as shown in Figure 3. Details on this set-up are given elsewhere [7]. In all experiments, fixed frequency radiation $(\lambda=266 \mathrm{~nm}$, SureLite I Nd:YAG [Continuum, Santa Clara, CA]) was used for non-resonant and near-resonant 2-photon ionization of $\mathrm{NO}$ and benzene, respectively. Ions were detected using two cascaded multichannel plates and fast data acquisition electronics (Bruker B500 digitizer/ Motorola [Tempe, AZ] MVME 68060 based VME bus computer combination).

The carrier and dilution gases helium 5.0 and ultra pure synthetic air were obtained from Oxygen Service Co. (St. Paul, MN) and used without further purification. Premixed NO/air gas (1\%) and pure NO were obtained from Air Gas (Radnor, PA) and used directly. Benzene (Aldrich [St. Louis, MO] $>99 \mathrm{vol} \%$ ) was purified in several pump-freeze-degassing cycles. The vapor was diluted with He. The analyte 

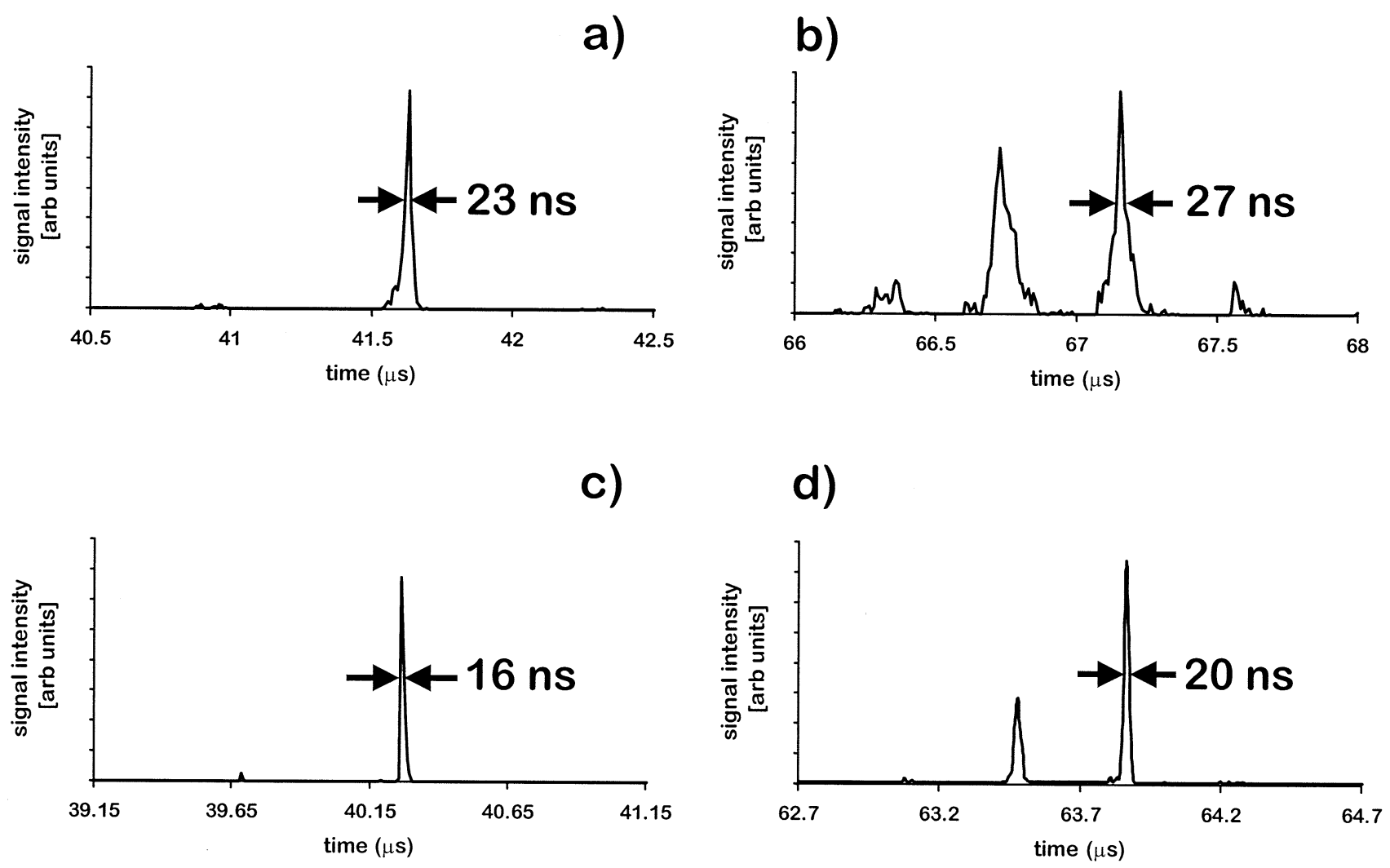

Figure 4. Comparison of resolution measurements obtained with the all stainless steel reflectron (a), (b), and the hybrid polymeric/stainless steel reflectron (c), (d). Mass spectra of benzene presented in plots (b) and (d) show parent ion resolutions of $t / 2 \Delta t=1200$ and 1500, respectively. Mass spectra of $\mathrm{NO}$ are presented in plots (a) and (c) and show resolutions of $t / 2 \Delta t=900$ and 1200 , respectively.

gases were diluted using MKS mass flow controllers and a down-stream pressure regulator, as described in detail in [6].

\section{Results}

A series of experiments were performed with $\mathrm{NO}$ and benzene to compare the resolution of a gridless dual stage hybrid reflectron comprised of a Vespel SP-22 second stage and a classical stainless steel ring first stage with an all stainless steel reflectron. Figure 4 presents mass spectra of $\mathrm{NO}$ and benzene obtained with the classical assembly (Figure a and b), and the hybrid reflectron (Figure $\mathrm{c}$ and $\mathrm{d}$ ). All spectra are the sum of 100 laser shots. For benzene, the parent ion and its surrounding fragments are shown while only mass 30 is shown for NO. Due to the smaller aperture used in the hybrid reflectron, higher voltages were required as compared to the classical assembly. This results in shorter flight times but does not adversely affect the resolution attained. When incorporating the classical reflectron, the mass resolution, expressed as $R=t / 2 \Delta t$, calculated by measuring the full width at half the peak maximum (FWHM), is 900 for nitric oxide and 1200 for the parent ion of benzene. The mass resolution achieved using the conductive polymer electrode is 1200 for nitric oxide and 1500 for the parent ion of benzene. Although little qualitative information can be gained from these resolution measurements, it is clearly evident that a reflectron partially composed of a carbon-filled polyimide electrode has at least equivalent resolving powers to one composed solely of discrete metallic electrodes. It is pointed out that the switching cycles do not lead to any noticeable artifacts from electronic noise in the recorded mass spectra.

\section{Conclusions}

The application of carbon-filled polyimides, in particular DuPont's Vespel SP-22, as novel conductive electrodes for use in ion optical devices and specifically in time-of-flight mass spectrometry has been presented. Preliminary results comparing resolution data indicate that these electrode performs at least as well as the traditional resistively coupled stacked electrode approach to reflectron design. However, due to the uniform nature of the electric field produced with this type of electrode [15], we believe that a reflectron composed entirely of conductive polymeric elements sandwiched between metallic electrodes would be superior to a discrete metal electrode stack design.

Ongoing research with graphite fiber filled polyimide (DuPont) and polyetheretherketone (RTP) has enabled the construction of volume conductive electrodes. 
The inside and outside dimensions of these electrodes can be machined to obtain either a linear or a non-linear electrical field. The use of volume conductive electrodes in all facets of charged particle optics, including lenses, ion sources, and reflectrons, is currently being explored. Particularly intriguing is the generation of "ideal" methods for ion energy focusing. Here, an ion source and/or reflectron is constructed so that the voltage at any point $\mathrm{x}$ approximates $\mathrm{V}_{\mathrm{x}}=\mathrm{ax}^{2}$, producing a field in which the time of flight of an ion is dependent only upon the square root of the mass and therefore independent of kinetic energy [18].

Another application of carbon filled conductive electrodes is in coaxial multi-pass TOF MS designs. In these instruments, a high mass resolution can be obtained by pulsing the potential over an assembly of electrodes connected through a bleeder network [19, 20]. However, electric ringing effects limit the minimum rise or fall time of such a network. One approach to circumvent this oscillatory behavior is to replace the resistive bleeder network with a matched RC network. Critically damped ringing-free networks with a response time of 50 ns have been reported [20]. When replacing the stack of discrete electrodes with a single bulk electrode, ringing effects are expected to be much less prominent. Response times of these materials well below 1 ns have been documented [15]. However, due to the specifications of commercially available high voltage switching components, the minimum expected rise/fall times of instruments made with these polymeric electrodes is limited to $5 \mathrm{~ns}$. Rise/fall times on this time scale have been observed in our laboratory and will be presented in an upcoming publication.

\section{Acknowledgments}

This work was supported by the NSF Atmospheric Program, grant no. 0083436 , and in part by the BMBF, Germany, within projects 01 LO 950/6 and 07 AK 302/0.

\section{References}

1. Pillay, A. E. A Review of Accelerator-Based Techniques in Analytical Studies. J. Radioanal. Nucl. Chem. 2000, 243, 191-197.

2. Mamyrin, B. A. Time-of-Flight Mass Spectrometry (Concepts, Achievements, and Prospects). Int. J. Mass Spectrom. 2001, 206, 251-266.

3. Gruen, D. M.; Young, C. E.; Pellin, M. J. Photo Ion Spectrometer. U.S. Patent no. $4,855,596$.
4. Even, U.; Dick, B. Computer Optimization for High-Resolution Time-of-Flight Mass Spectrometer. Rev. Sci. Instr. 2000, 71, 4415-4420.

5. McKeachie, J. R.; van der Veer, W. E.; Short, L. C.; Garnica, R. M.; Appel, M. F.; Benter, T. Selective Ultra-Trace Detection of $\mathrm{NO}$ and NO2 in Complex Gas Mixtures Using BroadBandwidth REMPI Mass Spectrometry. Analyst 2001, 126, 1221-1228.

6. Garnica, R. M.; Appel, M. F.; Eagan, L.; McKeachie, J. R.; Benter, T. A REMPI Method for the Ultrasensitive Detection of $\mathrm{NO}$ and NO2 Using Atmospheric Pressure Laser Ionization Mass Spectrometry. Anal. Chem. 2000, 72, 5639-5646.

7. Schmidt, S.; Appel, M. F.; Garnica, R. M.; Schindler, R. N.; Benter, T. Atmospheric Pressure Laser Ionization. An Analytical Technique for Highly Selective Detection of Ultralow Concentrations in the Gas Phase. Anal. Chem. 1999, 71, 37213729.

8. Hopkins, A. R.; Lewis, N. S. Detection and Classification Characteristics of Arrays of Carbon Black/Organic Polymer Composite Chemiresistive Vapor Detectors for the Nerve Agent Simulants Dimethylmethylphosphonate and Diisopropylmethylphosponate. Anal. Chem. 2001, 73, 884-892.

9. Skotheim, T. A.; Elsenbaumer, R. L.; Reynolds, J. R., Eds. Handbook of Conducting Polymers. Marcel Dekker, Inc: New York, 1998; 2nd ed. 963.

10. "Vespel Data Sheet", DuPont.

11. Goembel, L.; Doering, J. P. A Compact, Light Weight, High Resolution Electron Monochromator. Rev. Sci. Instr. 1995, 66, 3472-3474.

12. Tang, H.; Feng, H. Q.; Luo, H. B.; Dong, L. S.; Feng, Z. L. The Aggregation State of Polyimide. Eur. Polym. J. 1997, 33, 519523.

13. Hait, P. W. The Application of Polyimide to Ultrahigh Vacuum Seals. Vacuum (UK) 1967, 17, 547-550.

14. Nagashima, A.; Nishitani, T.; Fujisawa, T.; Sugie, T.; Kasai, S. Development of Vacuum Seals for Diagnostic Windows of the International Thermonuclear Experimental Reactor. Rev. Sci. Instr. 1999, 70, 460-463.

15. Sichel, E. K., Ed. Carbon Black-Polymer Composites: The Physics of Electrically Conducting Composites, Vol. III. Marcel Dekker, Inc: New York, 1982, 79.

16. Ghosh, P.; Chakrabarti, A. Conducting Carbon Black Filled EDPM Vulcanizates: Assessment of Dependence of Physical and Mechanical Properties and Conducting Character on Variation of Filler Loading. Eur. Polym. J. 2000, 36, 1043-1054.

17. Dahl, D. A. Simion 7.0; Ion Source Software: P.O. Box 2726, Idaho Falls, ID 83403.

18. Cotter, R. J. Time-of-Flight Mass Spectrometry: Instrumentation and Applications in Biological Research. American Chemical Society: Washington, DC, 1997, 60.

19. Piyadasa, C. K. G.; Hakansson, P.; Ariyaratne, T. R. A High Resolving Power Multiple Reflection Matrix-Assisted Laser Desorption/Ionization Time-of-Flight Mass Spectrometer. Rapid Commun. Mass Spectrom. 1999, 13, 620-624.

20. Park, M. Coaxial Multiple Reflection Time-of-Flight Mass Spectrometer. U.S. Patent no. 6,107,625. 